\title{
Reporting quality of randomized controlled trial abstracts published in leading laser medicine journals: An assessment using the CONSORT for Abstracts guidelines
}

\author{
Lu Jin $^{1,2} \quad$ Fang Hua $^{3 *}$ Qiang Cao ${ }^{1,2} *$ \\ ${ }^{1}$ State Key Laboratory of Military Stomatology \& National Clinical Research Center for Oral Diseases \& \\ Shaanxi Clinical Research Center for Oral Diseases, Department of Oral and Maxillofacial Surgery, \\ School of Stomatology, The Fourth Military Medical University, Xi'an, China \\ ${ }^{2}$ Medical Plastic and Aesthetic Center, Stomatological Hospital of The Fourth Military Medical \\ University, Xi'an, China \\ ${ }^{3}$ School of Dentistry, The University of Manchester, Manchester, United Kingdom \\ * Corresponding Authors: \\ Fang Hua, School of Dentistry, The University of Manchester, \\ JR Moore Building, Oxford Road, Manchester, M13 9PL, United Kingdom. \\ E-mail: drfanghua@outlook.com \\ Qiang Cao, Department of Oral and Maxillofacial Surgery, \\ School of Stomatology, The Fourth Military Medical University, \\ 145 West Changle Road, Xi'an, Shaanxi 710032, China. \\ E-mail: caoqiang921@hotmail.com
}

\section{How to Cite:}

Jin L, Hua F, Cao Q. Reporting quality of randomized controlled trial abstracts published in leading laser medicine journals: an assessment using the CONSORT for abstracts guidelines. Lasers in Medical Science. Epub ahead of print. doi:

$10.1007 / \mathrm{s} 10103-016-2018-4$

DOI: $10.1007 / \mathrm{s} 10103-016-2018-4$

PMID: 27365107

Please download the final published version from the publisher's website: http://link.springer.com/article/10.1007\%2Fs10103-016-2018-4 


\title{
Reporting quality of randomized controlled trial abstracts published in leading laser medicine journals: An assessment using the CONSORT for Abstracts guidelines
}

\begin{abstract}
The objectives of this study were to assess the reporting quality of randomized controlled trial (RCT) abstracts published in leading laser medicine journals, and investigate the association between potential predictors and reporting quality.
\end{abstract}

The official online archives of four leading laser medicine journals were hand-searched to identify RCTs published in 2014 and 2015. A reporting quality assessment was carried out using the original 16-item CONSORT (CONsolidated Standards Of Reporting Trials) for Abstracts checklist. For each abstract, an overall CONSORT score (OCS) was calculated (score range, 0 to 16). Univariable and multivariable linear regression analyses were performed to identify significant predictors of reporting quality. Chi-square (or the Fisher's exact) tests were used to analyze the adequate reporting rate of each quality item by specialty area.

A total of 129 RCT abstracts were included and assessed. The mean OCS was 4.5 (standard deviation, 1.3). Only three quality items (interventions, objective, conclusions) were reported adequately in most abstracts $(>80 \%)$. No abstract adequately reported results for the primary outcome, source of funding, and status of the trial. In addition, sufficient reporting of participants, outcome in the methods section, randomization, and trial registration was rare $(<5 \%)$. According to multivariable linear regression analysis, the specialty area of RCT abstracts was significantly associated with their reporting quality $(\mathrm{P}=0.008)$.

The reporting quality of RCT abstracts published in leading laser medicine journals is suboptimal. Joint efforts by authors, editors and other stakeholders in the field to improve trial abstract reporting are needed.

\section{Keywords}

Randomized controlled trials as topic, Lasers, Laser therapy, Medical writing, CONSORT, Abstracts 


\section{Introduction}

Complete, accurate and transparent reporting of health research is a vital aspect of translating findings into practice, and an ethical and moral responsibility of all researchers and other stakeholders [1]. In addition, a recent article indicated that at least $50 \%$ of biomedical research reports were sufficiently poor and therefore unusable, representing an avoidable waste of tens of billions of pounds [2]. Research into the current reporting quality and efforts to reduce relevant research waste are warranted.

In the era of evidence-based medicine, randomized controlled trials (RCTs) are considered the gold standard for assessing the effectiveness of healthcare interventions, and highest-grade evidence in the hierarchy of research designs [3]. But the quality of RCTs could be compromised by poor design and/or poor execution. Before applying the findings of an RCT into practice, clinicians need to critically appraise the RCT's reliability and applicability [4].

However, recent studies have shown that about $50 \%$ of biomedical research in general [5] and $60 \%$ of RCTs in dentistry [6] are behind the paywall. Healthcare professionals usually rely on abstracts to initially appraise a trial, decide whether or not to retrieve more information, or even directly inform their healthcare decision-making. Therefore, abstracts of RCTs should provide sufficient information about the trial and enable readers to carry out critical appraisals. [7]

In 2008, the CONSORT (CONsolidated Standards Of Reporting Trials) group released the CONSORT for Abstracts guidelines [7], providing guidance on the reporting of RCT abstracts in journals and conferences. However, according to recent studies, the reporting quality of RCT abstracts published in general medical journals [8] and specialty medical journals [9] remained suboptimal after the publication of CONSORT for Abstracts.

To our knowledge, there has been no assessment of the reporting quality of RCT abstracts in the field of laser medicine. Therefore, the objectives of the present study were to assess the reporting quality of recent RCT abstracts published in leading laser medicine journals, and to identify factors associated with abstract reporting.

\section{Methods}

\section{Selection of journals}

In this study, four journals which are mainly focused on the use of lasers in medical fields and have the highest impact factors in the 2014 Journal Citation Report [10] were included: Lasers in Surgery and Medicine (LSM; impact factor, 2.619), Lasers in Medical Science (LIMS; 2.489), Photomedicine and Laser Surgery (PLS; 1.672) and Journal of Cosmetic and Laser Therapy (JCLT; 1.110). 


\section{Collection of abstracts}

Two authors (L.J. and F.H.) hand-searched the included journals' official online archives, independently and in duplicate, to identify RCTs published in 2014 and 2015. Pre-specified inclusion criteria included: experimental research, human participants, health-care related interventions, presence of a control group, as well as random allocation of participants to interventions. When the eligibility of a study could not be determined with its title and abstract, the corresponding full-text was retrieved and checked. All discrepancies regarding eligibility were resolved by discussions among all authors. Thereafter, all included abstracts were collated into a Word document with journal title, author names and author affiliations removed to allow for blinded quality assessment. [9]

\section{Assessment of reporting quality}

Two authors (L.J. and F.H.) assessed the reporting quality of each included abstract independently and in duplicate, using the original CONSORT for Abstract guidelines and relevant explanations [7]. Any discrepancy was resolved through discussion. Among the 17 quality items of the original CONSORT for Abstract checklist, one item (authors) was designed specifically for conference abstracts and therefore excluded from our assessment. For each quality item, a score of " 1 " was given if the item was adequately reported, and a score of " 0 " if the reporting was inadequate. Then for each abstract, an overall CONSORT score (OCS; score range, 0 to 16 ) was calculated by totaling the scores of all the sixteen quality items. Additionally, we also documented the reporting of 11 sub-items of applicable CONSORT items, as mentioned in the CONSORT for Abstracts explanations [7], to provide supplementary information.

\section{Data extraction}

From each included abstract, the following information was extracted by two authors (L.J. and F.H.), independently and in duplicate, as potential predictors of abstract reporting quality: journal title, publication date, specialty area (dentistry, dermatology, surgery, or other specialties), abstract structure (structured vs. unstructured), word count, sample size, significance of the main finding (positive vs. negative), provision of the exact $\mathrm{P}$ values (e.g. $\mathrm{P}=0.011$ rather than $\mathrm{P}<0.05$ ), number of authors, geographical origin (first author), number of affiliations (single vs. multiple), as well as the existence of international collaboration. In addition, from the corresponding full-text of each abstract, we also extracted the number of trial centers (single center vs. multi-center) and existence of any financial support. Discrepancies were resolved by discussion. Like in previous similar research [11], a positive 
main outcome was defined as a statistically significant difference between the study group and control group, or a stated preference for either treatment arm.

\section{Statistical analyses}

Descriptive statistics were used to summarize the overall reporting quality and the reporting of each quality item/sub-item. Chi-square (or the Fisher's exact) tests were used to analyze the adequate reporting rate of each item by specialty area. In addition, we performed linear regression analyses to determine the association between potential predictors and abstract reporting quality (dependent variable, OCS). Univariable analyses were conducted first, and then all significant predictors in the univariable analyses were entered individually into a multivariable analysis. No significant violation of normality was found in assessments of the residuals. During multivariable modeling, tolerance and the variance inflation factor (VIF) were used to detect multicollinearity. Any predictor with a tolerance below 0.1 and/or a VIF above 10 was excluded from the final model [12]. For all analyses, the statistical significance level was set at $\mathrm{P}<0.05$.

\section{Results}

\section{Characteristics of included abstracts}

During the hand-search, a total of 129 RCT abstracts were deemed eligible and included. As demonstrated in Table 1, most abstracts were published in LIMS (46.5\%) or PLS (27.1\%), presenting single center trials $(95.3 \%)$, and with a positive main finding (81.4\%). Among medical specialties, dentistry (39.5\%) contributed the highest number of RCTs, followed by dermatology (26.4\%) and surgery (18.6\%). About half of the abstracts were written in 200 to 250 words $(47.3 \%)$ and in a structured format $(52.7 \%)$.

\section{Reporting of general items}

Table 2 shows the overall adequate reporting rate of each CONSORT item and sub-item in the included abstracts. Among the 129 abstracts included, only 60 (46.5\%) can be identified as randomized through their titles. Only $26(20.2 \%)$ stated explicitly the trial design (e.g. parallel, crossover, split-mouth). None of the abstracts reported funding sources. Only 4 abstracts (3.1\%) provided details about trial registration.

\section{Reporting of trial methodology}

The quality items objective (98.4\%) and interventions (80.6\%) were adequately reported by most abstracts. However, only 6 abstracts (4.7\%) clearly defined the primary outcome of the trial. In terms of information about trial participants, although $76.7 \%$ of the abstracts provided 
the eligibility criteria, only $2.3 \%$ described the setting in which participants were studied. In addition, $13.2 \%$ of the abstracts did not mention random assignment. Only $2(1.6 \%)$ and 1 $(0.8 \%)$ of the included abstracts described the methods used for sequence generation and allocation concealment, respectively. Forty-one abstracts (31.8\%) provided information about blinding (also referred to as masking), but only 14 (10.9\%) described explicitly who (e.g. participants, outcome assessors) was blinded.

\section{Reporting of trial results}

About $60 \%$ of the included abstracts reported the number of participants randomized to each group, but only $7 \%$ provided the number of participants analyzed in each group. Only 1 abstract $(0.8 \%)$ stated the adoption of per-protocol or intention-to-treat analysis. In addition, of those six abstracts that defined the primary outcome in their methods sections, no one provided the result of primary outcome in each group, the corresponding effect size or its precision. Besides, only 28 abstracts $(21.7 \%)$ described whether there was any adverse events or side effects.

\section{Reporting of trial conclusion}

Almost all the included abstracts (97.7\%) stated conclusions that were consistent with the trial results. However, only the conclusions of 15 abstracts (11.6\%) balanced the benefits and harms.

\section{Overall CONSORT score and associated factors}

The mean overall CONSORT score (OCS) of the included 129 abstracts was 4.53 (SD, 1.32; $95 \%$ CI, 4.30 to 4.76 ).

Table 3 shows the results of linear regression analyses. According to univariable analyses, a greater OCS was significantly associated with a structured format $(\mathrm{P}<0.001)$, greater word count ( $\mathrm{P}=0.034)$, a topic in dermatology $(\mathrm{P}<0.001)$, being published in the LSM $(\mathrm{P}<0.001)$, provision of the exact $\mathrm{P}$ value $(\mathrm{P}=0.019)$, and a first author from North America $(\mathrm{P}=0.018)$. However, in the multivariable analysis, journal of publication was excluded due to significant multicollinearity $(\mathrm{VIF}>10)$. Five predictors were therefore entered into the final model $\left(\mathrm{P}=0.001 ; \mathrm{R}^{2}, 0.226\right.$; adjusted $\left.\mathrm{R}^{2}, 0.160\right)$, among which only specialty remained a significant predictor of the OCS ( $B=0.83 ; 95 \% \mathrm{CI}, 0.22$ to $1.44 ; \mathrm{P}=0.008)$.

\section{Reporting quality in each specialty area}

Fig. 1 illustrates the reporting of each quality item and sub-item by specialty areas. According to chi-square (and Fisher's exact) tests, the adequate reporting rate of 4 CONSORT items and 
3 sub-items were significantly different among abstracts from different specialty areas: trial design ( $\mathrm{P}=0.007)$, settings $(\mathrm{P}=0.021)$, blinding $(\mathrm{P}=0.010)$, generic blinding only $(\mathrm{P}=0.018)$, number analyzed $(\mathrm{P}=0.034)$, harms $(\mathrm{P}<0.001)$, and balanced conclusion $(\mathrm{P}<0.001)$. Among specialty areas, abstracts from dermatology had the best performance for most of these items/sub-items.

\section{Discussions}

\section{Overall reporting quality}

Based on an assessment of 129 recently published RCT abstracts, the results of this study suggest that the current reporting quality of RCT abstracts in leading laser medicine journals is suboptimal. Of the 16 original CONSORT items, only 3 (interventions, objective, conclusions) were reported in more than $80 \%$ of the included abstracts, whereas 3 items (recruitment, outcome in the results section, funding) were never adequately reported. In addition, less than $5 \%$ of the abstracts provided sufficient information for participants, outcome in the methods section, randomization, and trial registration. This pattern of inadequate reporting is generally in line with findings of several previous studies in dentistry $[9,13,14]$ and other specialties [15-17], and indicates a lack of awareness of the CONSORT for Abstracts guidelines among researchers in the filed of laser medicine.

\section{Reporting of items related to RCT identification}

RCTs are the best primary studies to inform healthcare decisions and also form the foundation of systematic reviews and clinical guidelines. Identifiability of RCTs is thus vital for the completeness and quality of the evidence base. The Cochrane Highly Sensitive Search Strategy (Cochrane HSSS), which has been widely used by systematic reviewers to retrieve RCT reports since 1994, is mainly composed of controlled vocabulary (e.g. MeSH terms) and free-text terms related to the word "random" and specific trial designs (e.g. cross-over) [18]. Further considering that most databases do not contain the full-texts of indexed studies, it is crucial that RCT abstracts include words that facilitate correct indexing and be identifiable to common search strategies such as the Cochrane HSSS.

However, in this study, $54 \%$ of the included abstracts cannot be identified as randomized through their titles, and $80 \%$ did not describe explicitly the trial design. Furthermore, as high as $13 \%$ of the abstracts did not mention the word "random" at all in either the title or the body of abstract. Only after scrutiny of their full-texts were we able to identify these studies as RCTs. Such reporting deficiencies can result in inappropriate database indexing [19] and biases in systematic reviews [18]. 


\section{Reporting of items related to biases in RCTs}

The reliability of the findings of an RCT depends on the extent to which potential biases have been avoided. Inadequate allocation concealment and inappropriate sequence generation may lead to systematic differences between groups at baseline (selection bias). If participants and/or the personnel are not blinded, the trial results may be influenced by factors other than the interventions of interest (performance bias). Similarly, if outcome assessors and/or analysts are not blinded, the outcome measurement and/or analyses may be unreliable (detection bias). In addition, a high proportion or unbalanced distribution of dropouts suggests that there may be systematic differences between the dropouts in each group (attrition bias). When the pre-specified primary outcome(s) of a trial is not reported, the trial is at high risk of selective reporting bias. [20,21] Besides, if a trial is funded by the industry, its results could be biased in favor of the sponsor $[22,23]$.

Among the included abstracts of this study, however, CONSORT items related to the abovementioned sources of bias were either unreported (funding) or rarely reported (allocation concealment, sequence generation, blinding, numbers analyzed, outcome, trial registration). This suggests that the current reporting quality of most RCT abstracts in laser medicine does not allow readers to carry out adequate, initial critical appraisal of the trials.

\section{Reporting of items related to the applicability of findings}

Readers of an RCT need a description of the trial participants and the setting in which participants were studied to determine the generalizability of the trial to their own setting. [7,21] Also, readers need information about adverse events and side effects occurred in a trial to make rational and balanced decisions. [7]

In this study, although most abstracts provided the eligibility criteria (e.g. demographics, clinical diagnosis) of participants, only several described the study setting (e.g. primary, secondary care). Besides, only about one fifth of the abstracts stated whether there were any adverse events, and only one tenth balanced the benefits and harms in their conclusions.

\section{Factors associated with abstract reporting}

In univariable analyses, we found that specialty area, journal of publication, word count, abstract structure, continent of origin and provision of exact $P$ values were associated with the reporting quality of RCT abstracts. These findings are generally in line with previous studies in dentistry [9,11,13], dermatology [24], and oncology [15,25].

However, in the multivariable analysis, only specialty area remained a significant predictor of reporting quality. Chi-square tests also indicate that, for certain quality items/sub-items, abstracts from different specialties had significantly different reporting 
quality. This finding suggests that abstract reporting behavior varies among researchers from different specialty areas/research fields. When taking measures to improve the reporting in a certain field, stakeholders (e.g. editors, educators, funders) need to take into consideration the specific reporting problems and information needs of researchers in that field.

\section{Limitations and strengths}

Our study is not without limitations. Firstly, we only included four SCIE-indexed, high-impact journals in the field of laser medicine, thus our results may not be generalizable to RCT abstracts published in other laser medicine or general medical journals. However, using abstracts published in high-impact, SCIE-indexed journals as the materials of assessment is a method commonly used in previous similar research $[8,9,14,26]$. Second, with multivariable linear regression analysis we found that specialty area is a significant predictor of reporting quality. But our final model can only explain $16 \%$ (adjusted $\mathrm{R}^{2}$ ) to $23 \%\left(\mathrm{R}^{2}\right)$ of the variation of OCS. Nevertheless, a total of 14 potential factors have been investigated in the present study, which is already more than other similar studies [9,13,15,25]. Roles of other potential predictors of abstract reporting quality could be investigated in future research.

Despite these limitations, the present study has several strengths. To our knowledge, this is the first study regarding reporting quality in the field of laser medicine, and the first of its kind to explore the association between specialty area of abstracts and their reporting quality. In addition, the original CONSORT for Abstracts guidelines were used for quality assessment.

\section{Implications}

Sufficient reporting of health research is a responsibility of all authors, editors, funders and other stakeholders [1]. When writing RCT abstracts, authors should make full use of the space allowed by journals, and report as much key information as possible according to the CONSORT for Abstracts guidelines [7].

In addition, a previous study showed that endorsement of the CONSORT for Abstracts guidelines in "instructions to authors', combined with active policies to implement these guidelines, had led to a significant improvement of RCT abstract reporting in leading medical journals [27]. We recommend that journal editors in the field of laser medicine also consider endorsing the CONSORT for Abstracts guidelines and other reporting guidelines (available at the EQUATOR Network: www.equator-network.org) relevant to their journals [28]. Such efforts are likely to not only improve the quality of research reporting, but also help reduce relevant avoidable research waste in the field of laser medicine [2]. 


\section{Conclusions}

- The reporting quality of RCT abstracts published in leading laser medicine journals is suboptimal;

- The specialty area of RCT abstracts are significantly associated with their reporting quality;

- Joint efforts by authors, editors and other stakeholders in the field to improve the reporting of RCT abstracts are needed. 


\section{References}

1. Needleman I, Moher D, Altman DG, Schulz KF, Moles DR, Worthington H (2008) Improving the clarity and transparency of reporting health research: a shared obligation and responsibility. Journal of dental research $87(10): 894-895$

2. Glasziou P, Altman DG, Bossuyt P, Boutron I, Clarke M, Julious S, Michie S, Moher D, Wager E (2014) Reducing waste from incomplete or unusable reports of biomedical research. Lancet 383 (9913):267-276. doi:10.1016/S0140-6736(13)62228-X

3. Concato J, Shah N, Horwitz RI (2000) Randomized, controlled trials, observational studies, and the hierarchy of research designs. The New England journal of medicine 342 (25):1887-1892. doi:10.1056/NEJM200006223422507

4. Richards D (2009) Critically appraising randomised trials. Evidence-based dentistry 10 (3):88-90. doi:10.1038/sj.ebd.6400673

5. Kurata K, Morioka T, Yokoi K, Matsubayashi M (2013) Remarkable growth of open access in the biomedical field: analysis of PubMed articles from 2006 to 2010. Plos one 8 (5):e60925. doi:10.1371/journal.pone.0060925

6. Hua F, Sun H, Walsh T, Worthington H, Glenny AM (2016) Open access to journal articles in dentistry: Prevalence and citation impact. Journal of dentistry 47:41-48. doi:10.1016/j.jdent.2016.02.005

7. Hopewell S, Clarke M, Moher D, Wager E, Middleton P, Altman DG, Schulz KF, Group C (2008) CONSORT for reporting randomized controlled trials in journal and conference abstracts: explanation and elaboration. Plos Med 5 (1):e20. doi:10.1371/journal.pmed.0050020

8. Ghimire S, Kyung E, Kang W, Kim E (2012) Assessment of adherence to the CONSORT statement for quality of reports on randomized controlled trial abstracts from four high-impact general medical journals. Trials 13:77. doi:10.1186/1745-6215-13-77

9. Hua F, Deng L, Kau CH, Jiang H, He H, Walsh T (2015) Reporting quality of randomized controlled trial abstracts: survey of leading general dental journals. Journal of the American Dental Association (1939) 146 (9):669-678.e661. doi:10.1016/j.adaj.2015.03.020

10. Thomson Reuters (2015) 2014 Journal Citation Reports ${ }^{\circledR}$ Science Edition. https://jcr.incites.thomsonreuters.com/JCRJournalHomeAction.action. Accessed 11 January 2016

11. Hua F, Walsh T, Glenny AM, Worthington H (2015) Reporting quality of randomized controlled trial abstracts presented at European Orthodontic Society congresses. European journal of orthodontics. doi:10.1093/ejo/cjv094

12. Norman G, Streiner D (2008) Biostatistics: The Bare Essentials. 3rd ed edn. BC Decker Inc., 
Hamilton, Ontario

13. Fleming PS, Buckley N, Seehra J, Polychronopoulou A, Pandis N (2012) Reporting quality of abstracts of randomized controlled trials published in leading orthodontic journals from 2006 to 2011. American journal of orthodontics and dentofacial orthopedics : official publication of the American Association of Orthodontists, its constituent societies, and the American Board of Orthodontics 142 (4):451-458. doi:10.1016/j.ajodo.2012.05.013

14. Faggion CM, Jr., Giannakopoulos NN (2012) Quality of reporting in abstracts of randomized controlled trials published in leading journals of periodontology and implant dentistry: a survey. Journal of periodontology 83 (10):1251-1256. doi:10.1902/jop.2012.110609

15. Ghimire S, Kyung E, Lee H, Kim E (2014) Oncology trial abstracts showed suboptimal improvement in reporting: a comparative before-and-after evaluation using CONSORT for Abstract guidelines. Journal of clinical epidemiology 67 (6):658-666. doi:10.1016/j.jclinepi.2013.10.012

16. Cui Q, Tian JH, Song XP, Yang KH (2014) Does the CONSORT checklist for abstracts improve the quality of reports of randomized controlled trials on clinical pathways? Journal of evaluation in clinical practice 20 (6):827-833. doi:10.1111/jep.12200

17. Can OS, Yilmaz AA, Hasdogan M, Alkaya F, Turhan SC, Can MF, Alanoglu Z (2011) Has the quality of abstracts for randomised controlled trials improved since the release of Consolidated Standards of Reporting Trial guideline for abstract reporting? A survey of four high-profile anaesthesia journals. European journal of anaesthesiology 28 (7):485-492. doi:10.1097/EJA.0b013e32833fb96f

18. Robinson KA, Dickersin K (2002) Development of a highly sensitive search strategy for the retrieval of reports of controlled trials using PubMed. International journal of epidemiology 31 (1):150-153. doi:Doi 10.1093/lje/31.1.150

19. Wieland LS, Robinson KA, Dickersin K (2012) Understanding why evidence from randomised clinical trials may not be retrieved from Medline: comparison of indexed and non-indexed records. Bmj 344:d7501. doi:10.1136/bmj.d7501

20. Higgins JPT, Altman D, Sterne JAC (2011) Chapter 8: Assessing risk of bias in included studies. In: Higgins JPT, Green S (eds) Cochrane Handbook for Systematic Reviews of Interventions Version 5.1.0 [updated March 2011]. The Cochrane Collaboration,

21. Lewis SC, Warlow CP (2004) How to spot bias and other potential problems in randomised controlled trials. J Neurol Neurosur Ps 75 (2):181-187. doi:10.1136/jnnp.2003.025833

22. Bekelman JE, Li Y, Gross CP (2003) Scope and impact of financial conflicts of interest in biomedical research: a systematic review. Jama 289 (4):454-465

23. Lexchin J, Bero LA, Djulbegovic B, Clark O (2003) Pharmaceutical industry sponsorship and 
research outcome and quality: systematic review. Bmj 326 (7400):1167-1170. doi:10.1136/bmj.326.7400.1167

24. Dupuy A, Khosrotehrani K, Lebbe C, Rybojad M, Morel P (2003) Quality of abstracts in 3 clinical dermatology journals. Archives of dermatology 139 (5):589-593. doi:10.1001/archderm.139.5.589

25. Guo JW, Iribarren SJ (2014) Reporting quality for abstracts of randomized controlled trials in cancer nursing research. Cancer nursing 37 (6):436-444. doi:10.1097/NCC.0000000000000112

26. Chhapola V, Tiwari S, Brar R, Kanwal SK (2015) An interrupted time series analysis showed suboptimal improvement in reporting quality of trial abstract. Journal of clinical epidemiology. doi:10.1016/j.jclinepi.2015.10.013

27. Hopewell S, Ravaud P, Baron G, Boutron I (2012) Effect of editors' implementation of CONSORT guidelines on the reporting of abstracts in high impact medical journals: interrupted time series analysis. Bmj 344:e4178. doi:10.1136/bmj.e4178

28. Simera I, Moher D, Hirst A, Hoey J, Schulz KF, Altman DG (2010) Transparent and accurate reporting increases reliability, utility, and impact of your research: reporting guidelines and the EQUATOR Network. BMC medicine 8:24. doi:10.1186/1741-7015-8-24 


\section{Acknowledgements}

F.H. is a recipient of the President's Doctoral Scholar Award from The University of Manchester, but this study had no explicit funding. The funding source had no involvement in the study design, collection, analysis and interpretation of data, preparation of the manuscript, or in the decision to publish. 


\section{Tables}

Table 1. Characteristics of included abstracts

\begin{tabular}{|c|c|c|}
\hline Characteristic & Category & No. (\%) \\
\hline \multirow[t]{4}{*}{ Journal } & LSM & $22(17.1)$ \\
\hline & LIMS & $60(46.5)$ \\
\hline & PLS & $35(27.1)$ \\
\hline & JCLT & $12(9.3)$ \\
\hline \multirow[t]{2}{*}{ Year } & 2014 & $69(53.5)$ \\
\hline & 2015 & $60(46.5)$ \\
\hline \multirow[t]{4}{*}{ Specialty } & Dentistry & $51(39.5)$ \\
\hline & Dermatology & $34(26.4)$ \\
\hline & Surgery & $24(18.6)$ \\
\hline & Other & $20(15.5)$ \\
\hline \multirow[t]{5}{*}{ Continent } & Europe & $40(31.0)$ \\
\hline & Asia & $39(30.2)$ \\
\hline & South America & $32(24.8)$ \\
\hline & North America & $10(7.8)$ \\
\hline & Africa & $8(6.2)$ \\
\hline \multirow[t]{4}{*}{ Word count } & $<200$ & $7(5.4)$ \\
\hline & $200-250$ & $61(47.3)$ \\
\hline & $251-300$ & $41(31.8)$ \\
\hline & $>300$ & $20(15.5)$ \\
\hline \multirow[t]{2}{*}{ Structured format } & No & $61(47.3)$ \\
\hline & Yes & $68(52.7)$ \\
\hline \multirow[t]{2}{*}{ Exact $P$ value } & No & $88(68.2)$ \\
\hline & Yes & $41(31.8)$ \\
\hline \multirow[t]{2}{*}{ Trial outcome } & Negative & $24(18.6)$ \\
\hline & Positive & $105(81.4)$ \\
\hline \multirow[t]{2}{*}{ No. of centers } & Single center & $123(95.3)$ \\
\hline & Multi-center & $6(4.7)$ \\
\hline \multirow[t]{3}{*}{ No. of authors } & $<4$ & $24(18.6)$ \\
\hline & $4-7$ & $86(66.7)$ \\
\hline & $>7$ & $19(14.7)$ \\
\hline \multirow[t]{3}{*}{ Sample size } & $<25$ & $49(38.0)$ \\
\hline & $25-50$ & $43(33.3)$ \\
\hline & $>50$ & $37(28.7)$ \\
\hline \multirow[t]{2}{*}{ Funded } & No & $67(51.9)$ \\
\hline & Yes & $62(48.1)$ \\
\hline \multirow[t]{2}{*}{ Multiple affiliations } & No & $30(23.3)$ \\
\hline & Yes & 99 (76.7) \\
\hline \multirow[t]{2}{*}{ International collaboration } & No & $103(79.8)$ \\
\hline & Yes & $26(20.2)$ \\
\hline Overall & & $129(100.0)$ \\
\hline
\end{tabular}


Table 2. Reporting of each CONSORT checklist item and sub-item in the included 129 abstracts. [7]

\begin{tabular}{|c|c|c|}
\hline Items & Criteria and sub-items & No. (\%) \\
\hline 1. Title & Identification of the study as randomized & $60(46.5)$ \\
\hline 2. Trial design & Description of the trial design (e.g. parallel, cluster, crossover) & $26(20.2)$ \\
\hline \multirow[t]{3}{*}{ 3. Participant } & Eligibility criteria for participants and the settings where the data were collected & $2(1.6)$ \\
\hline & 3a. Eligibility criteria for participants & 99 (76.7) \\
\hline & 3b. Settings of data collection & $3(2.3)$ \\
\hline 4. Interventions & Interventions intended for each group & $104(80.6)$ \\
\hline 5. Objective & Specific objective or hypothesis & $127(98.4)$ \\
\hline 6. Outcome $1^{a}$ & Clearly defined primary outcome for this report & $6(4.7)$ \\
\hline \multirow[t]{4}{*}{ 7. Randomization } & How participants were allocated to interventions & $1(0.8)$ \\
\hline & 7a. Random assignment & $112(86.8)$ \\
\hline & 7b. Sequence generation & $2(1.6)$ \\
\hline & 7c. Allocation concealment & $1(0.8)$ \\
\hline \multirow[t]{2}{*}{ 8. Blinding (Masking) } & Whether or not participants, caregivers, and those assessing the outcomes were blinded & $14(10.9)$ \\
\hline & 8a. Generic description only (e.g. single-blind, double-blind) & 27 (20.9) \\
\hline 9. Numbers randomized & Number of participants randomized to each group & $77(59.7)$ \\
\hline 10. Recruitment & Trial status (e.g. on-going, closed to recruitment, closed to follow-up) & $0(0)$ \\
\hline \multirow[t]{2}{*}{ 11. Numbers analyzed } & Number of participants analyzed in each group & $9(7.0)$ \\
\hline & 11a. Intention-to-treat analysis or per-protocol analysis & $1(0.8)$ \\
\hline \multirow[t]{4}{*}{ 12. Outcome $2^{b}$} & For the primary outcome, a result for each group and the estimated effect size and its precision & $0(0)$ \\
\hline & 12a. Primary outcome result for each group & $0(0)$ \\
\hline & 12b. Estimated effect size & $0(0)$ \\
\hline & 12c. Precision of the estimate (e.g. Cl 95\%) & $0(0)$ \\
\hline 13. Harms & Important adverse events or side effects & $28(21.7)$ \\
\hline \multirow[t]{2}{*}{ 14. Conclusions } & General interpretation of the results & $126(97.7)$ \\
\hline & 14a. Benefits and harms balanced & $15(11.6)$ \\
\hline 15. Trial registration & Registration number and name of trial register & $4(3.1)$ \\
\hline 16. Funding & Source of funding & $0(0)$ \\
\hline
\end{tabular}

${ }^{\mathbf{a}}$ Outcome reported in Methods section; ${ }^{\mathbf{b}}$ Outcome reported in Results section. 
Table 3. Linear regression derived coefficients $(B)$ and $95 \%$ confidence intervals, with overall CONSORT score (OCS) as the dependent variable for the included 129 abstracts.

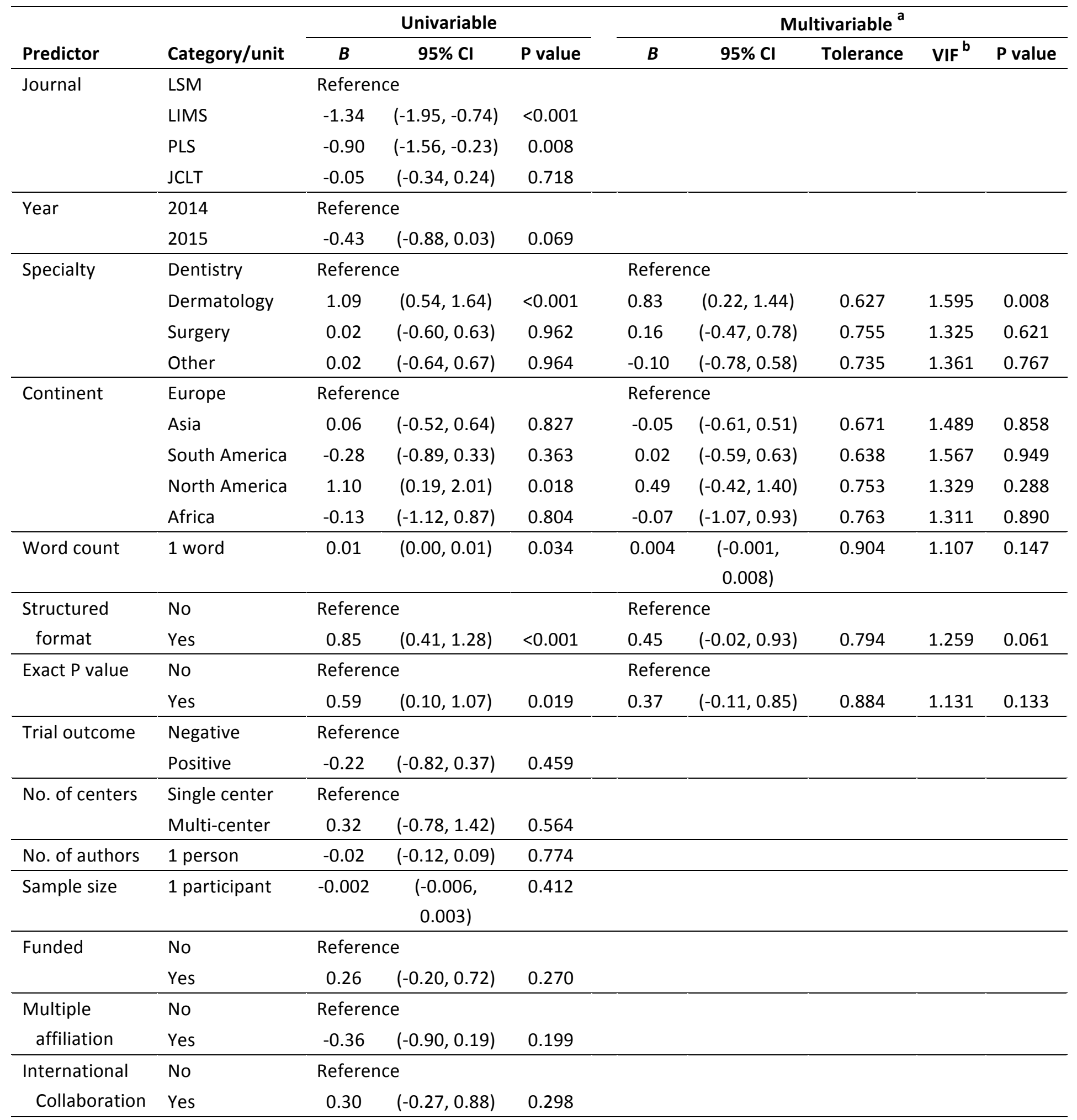

${ }^{a}$ For multivariable analysis, constant $=3.016, R^{2}=0.226$, adjusted $R^{2}=0.160, P=0.001$.

${ }^{\text {b }}$ VIF, variance inflation factor. 


\section{Figure}

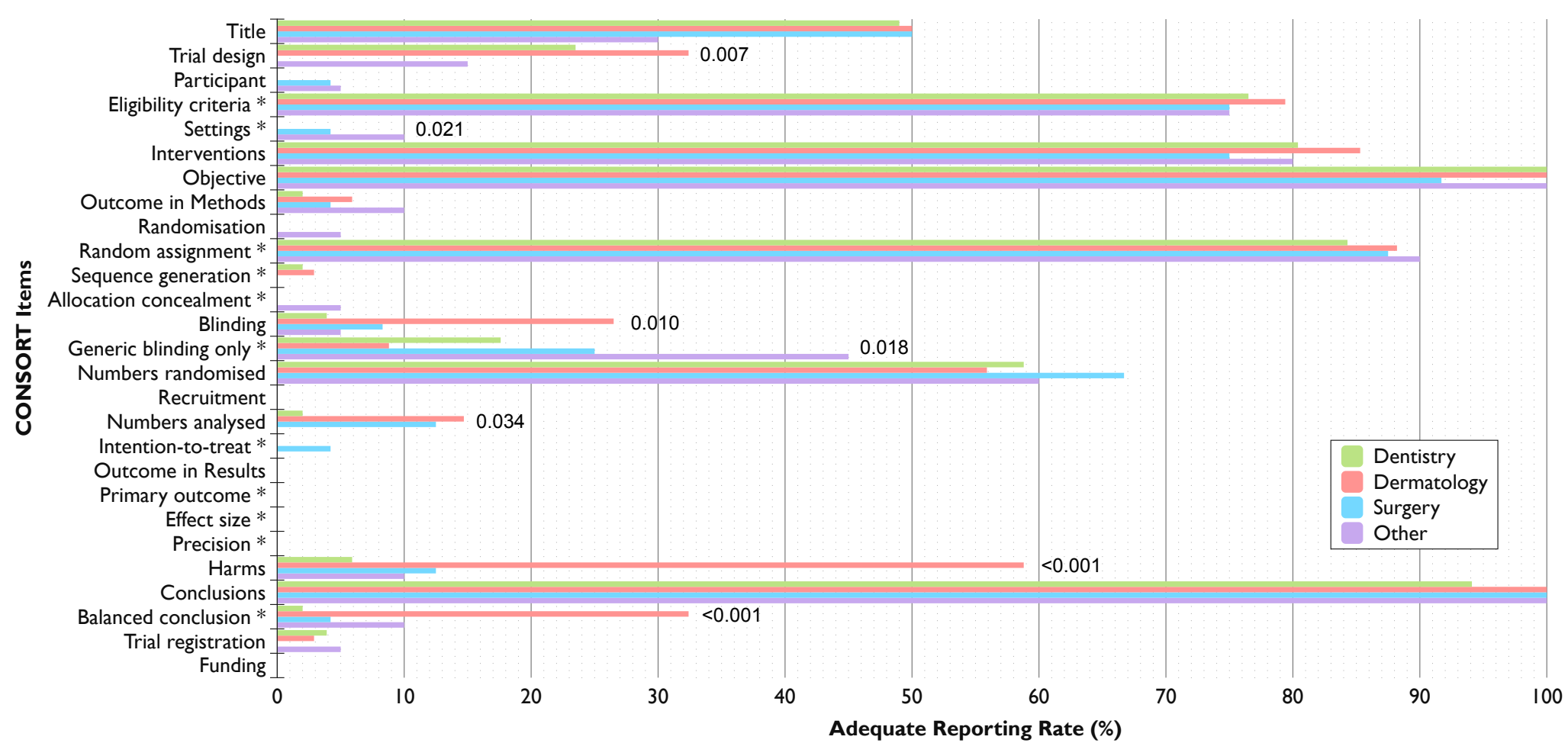

Fig. 1 Adequate reporting rate of each CONSORT item and sub-item by specialty. ${ }^{*}$ Sub-items of applicable CONSORT items. 